\title{
Resource Allocation for Uplink M2M Communication: A Game Theory Approach
}

\author{
Hashim Safdar, Norsheila Fisal, Rahat Ullah, Wajahat Maqbool, Faiz Asraf, Zubair Khalid, A.S. Khan
}

\author{
UTM-MIMOS, Center of Excellence for Telecommunication Technology \\ Faculty of Electrical Engineering, Universiti Teknologi Malaysia (UTM) \\ Skudai 81310, Johor, Malaysia
}

eng.hashim.s@ieee.org, sheila@fke.utm.my, \{eng.rahat.u, eng.wajahat.m\}@ieee.org, \{faiz.asraf, engr.zktanoli, adnan.ucit\}@gmail.com

\begin{abstract}
Machine-to-Machine (M2M) communication in cellular network is the driver for the future Internet of Things (IoT). The main challenge of M2M communication is the possibility of huge traffic in the uplink network that can cause problem in the network. This paper considers the problem of resource allocation among machines connecting in uplink to different femto base stations (FBSs). Resource allocation problem is analyzed through both non-cooperative and cooperative game to maximize their data rate and minimize utilization of power. Numerical result shows that by adapting non-cooperative game, all machines are getting data rate as per Nash Equilibrium (NE) or either they can set their strategy to maximize their data rate selfishly. On the other hand for coalitional game theory approach machines who participate in game are getting fair resource allocations.
\end{abstract}

Keywords- M2M communication; Resource allocation; Uplink; Game theory

\section{INTRODUCTION}

In recent years, field of wireless communication began to discuss the in-corporation among the machines in which meters, sensors and user equipment (smart phones) are involved [1]. Large coverage of wireless cellular network gives opportunity to communicate machines. It is assumed that millions of machines will connect to the cellular network directly or indirectly in the coming future $[1,2]$. Increase in numbers of machines which might be diverse in their nature is allowing industry to think about proper resource allocation and scheduling schemes to improve the network performance, which is normally not designed for M2M communication. M2M machines are using wireless uplink to communicate with base station (BS). If machine is under the coverage region of multiple BSs then it is likely to connect with the strongest or the nearest BS. In uplink communication resource allocation e.g. power is difficult to manage due to distributed power constraints of machines instead of centralized downlink communication [3], if all the machines are battery operated then total transmission power constraint is also an important issue.

For M2M, the main objective is to minimize their transmitting power and maximize their data rate. It is really hard to dedicate resources for each machine as the number of machine are increasing by time [2]. M2M communication typically transmitting short session from huge numbers of machines [4]. Random access might be a solution for huge numbers of machines to grab resources as it is hard to dedicate resources to each machine. Furthermore, the idea of cooperative machine grouping [5], along with a controller in between machine group and BS is discussed for energy efficient M2M communication [6]. Power efficient M2M communication system is evaluated by assigning equal distribution of minimum time and minimum bandwidth in time division multiple access (TDMA) and frequency division multiple access (FDMA) respectively [7]. Machines or a group of machines which have different data rate requirements then equal distribution of resources among them is not a fair solution. In single BS network, resources divided among machines are either by time or frequency. Furthermore, in single BS scenario, for efficient delay and channel aware resource allocation scheduling is a solution as discussed in [8].

In this paper, we take a new direction to analyze the resource allocation problem of $\mathrm{M} 2 \mathrm{M}$ communication under two femto base stations (FBSs) network with game theory. Recently, there has been significant research work that implements game theory for the analysis of resource allocation in wireless communication networks. This is basically due to the need for distributed mobile networks where machines can make independent and rational strategic decision [9]. Generally, game theory classified into two branches: noncooperative [10] and cooperative game theory [11]. Noncooperative game theory studies the strategic choices resulting from the interactions among competing players, where each player improving its strategy independently for improving its own performance or reducing its loss [12]. Nash equilibrium $(\mathrm{NE})$ is a one of the solution for non-cooperative game. Cooperative game theory provides analytical tools to study the behavior of rational players when they cooperate make a coalition to strengthen their positions in the game [11]. Access control by using cooperative game theory in multiple base station scenario discussed in [13] by putting uplink users in two coalition.

In this paper, we modeled a network FBSs with number of machines attached in uplink. Model is evaluated under both non-cooperative game and cooperative game theory techniques separately. In non-cooperative game, resource distribution falls to NE among all machines participating in this game. As the data rate requirement of machines is different and might be $\mathrm{NE}$ is not a fair solution.

The rest of paper is divided into following sections: Section II the system model and assumptions are presented. Section III and IV provide the analysis of the non-cooperative game 